\title{
The ferromagnetic to spin glass transition
}

\author{
A. Hamzić $\left({ }^{*}\right)$ and I. A. Campbell \\ Laboratoire de Physique des Solides, Université Paris-Sud, 91405 Orsay, France
}

(Reçu le 22 septembre 1980, accepté le 3 novembre 1980)

\begin{abstract}
Résumé. - A partir de mesures de magnétorésistance sur des alliages de $\mathrm{PtFe}$ et de $\underline{\mathrm{PdC}} \mathrm{Co}$, nous montrons qu'un désalignement au sein de l'état ferromagnétique se manifeste à des concentrations bien au-dessus de la concentration critique à laquelle l'ordre verre de spin s'installe. Pour le PtFe la transition ferromagnétique-verre de spin est de deuxième ordre.
\end{abstract}

\begin{abstract}
From magnetoresistance measurements in $\mathrm{PtFe}$ and $\mathrm{PdCo}$ alloys we show that disalignment within the ferromagnetic state begins at concentrations well above a critical concentration at which spin glass order sets in. In $\underline{\mathrm{PtFe}}$ the ferromagnetic to spin glass transition is second order.
\end{abstract}

A number of cases are known experimentally in which an alloy system at low temperature passes from a ferromagnetically ordered state to a spin glass state as the concentration of the components is varied. AuFe and PdMn are probably the best known examples [1, 2]. The initial low field susceptibility $\chi_{0}(T)$ has been by far the most widely used tool for determining transition temperatures and establishing phase diagrams.

We have made resistivity and magnetoresistance measurements on the alloy systems PtFe and PdCo. We have studied the variation of the local magnetic correlations with concentration in these alloys and we find that the transition from ferromagnet to spin glass as a function of concentration at $T=0$ is second order and that ferromagnetic order is broken down gradually over a wide concentration range.

We will first outline the principle of the method. We will consider a dilute magnetic alloy in which the local moment $S$ on each magnetic atom is well defined, in the sense that we will ignore any possible Kondo effect complications. The magnetic atoms interact with the conduction electrons through $V+J \mathbf{S . s}$; in the high temperature paramagnetic state with applied field zero, the total scattering is [3]

$$
\rho_{\mathrm{m}}=k c\left(V^{2}+J^{2} S(S+1)\right)
$$

where $k$ is a constant and $c$ is the concentration. As

(*) Permanent address : Institute of Physics of the University, Zagreb, Yugoslavia. the temperature is lowered and the spins become frozen through interactions, the inelastic scattering $k c J^{2} S$ is frozen out, and the elastic disorder scattering may or may not be reduced depending on the type of ordered state into which the system freezes. The elastic scattering will be [3]

$$
k c\left(V^{2}+J^{2} S^{2}-4\left(J^{2}\langle M\rangle_{\lambda}^{2}\right) /\left(1+J^{2} S^{2} / V^{2}\right)\right)
$$

where $\langle M\rangle_{\lambda}$ is the average magnetization per atom taken over a region of dimensions of the electronic mean free path $\lambda$. At $T=0$ there is no inelastic scattering and we will write $\langle M\rangle_{\lambda}^{0}$ for $\langle M\rangle_{\lambda}$ at zero temperature and in zero applied field.

We have three possible situations :

i) If the low temperature state is a true ferromagnet where all the moments are parallel, $\langle M\rangle_{\lambda}^{0}=S$ and the scattering is reduced to a minimum at $T=0$.

ii) If the low temperature state is a spin glass, $\langle M\rangle_{\lambda}^{0}=0$ and the elastic scattering is as high at $T=0$ as in the paramagnetic state, so the elastic scattering is independent of temperature.

iii) Finally, a range of intermediate situations are possible in which there is a local average magnetization at $T=0$ but where the spins are not fully aligned, so $0<\langle M\rangle_{\lambda}^{0}<S$. This we will call a quasiferromagnet. The elastic scattering will be reduced compared to the paramagnetic state but will not be as small as if the spins were fully aligned.

Now if a magnetic field is applied, it will have no effect on the resistivity of a true ferromagnet at $T=0$ (the local order within each domain is already 
maximum). For a spin glass or quasiferromagnet on the other hand even at $T=0$ there will be a negative magnetoresistance, saturating at a field sufficient to fully align the spins. The ratio of this saturation magnetoresistance to the total change in resistance associated with full magnetization (see Fig. 1) will be

$$
\rho_{\mathrm{sat}} / \rho_{\mathrm{m}}=1-\left(\langle M\rangle_{\lambda}^{0} / S\right)^{2} .
$$

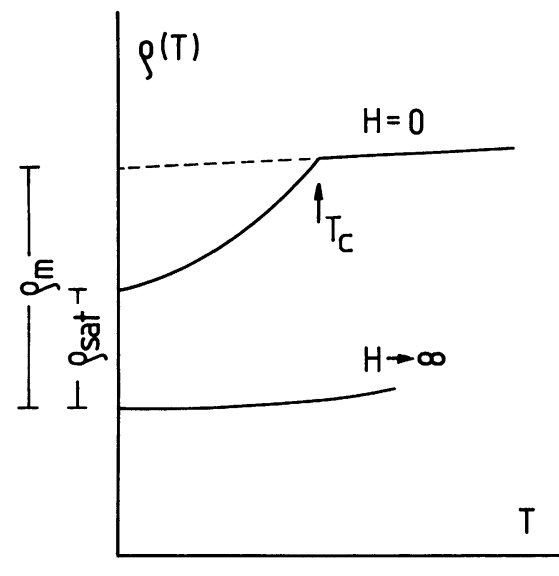

Fig. 1. - The temperature dependence of the resistivity of a quasiferromagnet at zero and at high field (schematic).

The resistivity technique has the advantage over direct magnetic measurements of allowing us to estimate the local magnetization within a domain in zero applied field.

As an example, experimental data for $\mathrm{PtFe} 1.1 \%$ are shown in figure 2. (Corrections have been made

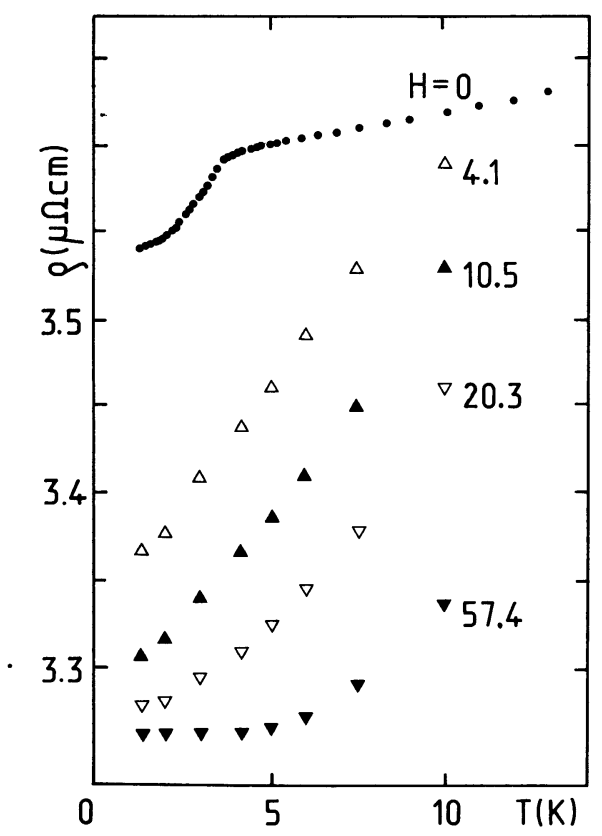

Fig. 2. - The temperature dependence of the resistivity of $\mathrm{Pt}$ $1.1 \% \mathrm{Fe}$ at zero field and in fields of $4.1,10.5,20.3$ and $57.4 \mathrm{kG}$. Small ordinary magnetoresistance corrections have been made. for the ordinary magnetoresistance, which is weak at this concentration.) We can estimate

$$
\rho_{\mathrm{sat}} / \rho_{\mathrm{m}}=0.90
$$

The concentration dependence of $\left(1-\rho_{\text {sat }} / \rho_{\mathrm{m}}\right)$, i. e. of $\left(\langle M\rangle_{\lambda}^{0} / S\right)^{2}$, for $\underline{\mathrm{PtFe}}$ and $\underline{\mathrm{PdCl}}$ alloys is shown in figures 3 and 4 . Both these systems have been considered good ferromagnets, with Curie temperatures of about $20 \mathrm{~K} / \%$ and $40 \mathrm{~K} / \%$ respectively above $1 \%$ concentration [4]. The present results show that for both systems when $c>5 \%$, the ferromagnetic order is indeed almost complete (zero magnetoresistance as $T \rightarrow 0$ ) but as the concentration is lowered the zero field ferromagnetic order breaks down gradually and the spins become more and more disaligned. For PtFe at $c \simeq 0.9 \%$ there is a transition to a spin glass state [5] $\left(\rho_{\text {sat }} / \rho_{\mathrm{m}}=1\right)$. A very similar critical concentration has been estimated for this system from magnetic measurements [6] but no indication of the gradual breakdown of the ferromagnetic state was obtained from the magnetic data.

For the PdCo the disaligning process is much more gradual and a spin glass state has not yet set in at

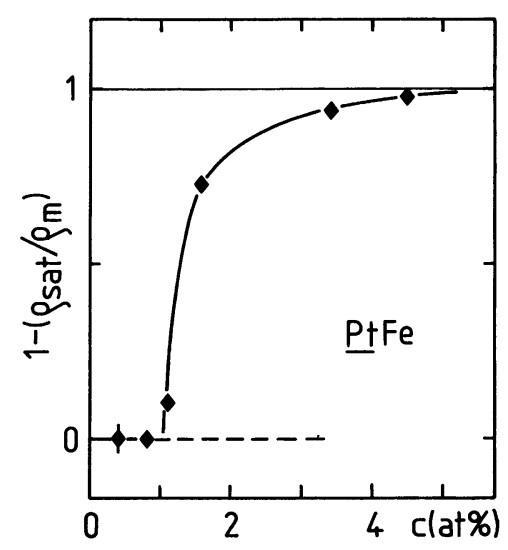

Fig. 3. - The observed variation with concentration of the parameter $1-\left(\rho_{\text {sat }} / \rho_{\mathrm{m}}\right)$ in PtFe alloys.

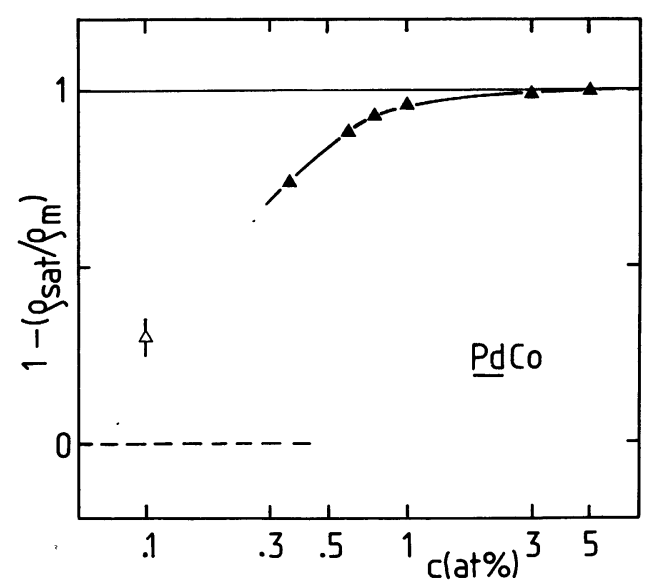

Fig. 4. - The variation with concentration of the parameter $1-\left(\rho_{\text {sat }} / \rho_{\mathrm{m}}\right)$ in PdCo alloys. $\Delta$ present results, $\Delta$ reference [7]. 
$c=0.1 \%$ [7], despite the fact that some disalignment was already visible at a concentration fifty times higher. Measurements at lower concentrations and at low temperature will be necessary to determine if a transition actually takes place at $\sim 0.05 \% \mathrm{Co}$, as an extrapolation of the present data would indicate (see also Ref. [18]).

It is clear from figure 3 that if we take $\langle M\rangle_{\lambda}^{0}$ as the order parameter, the transition as a function of the concentration in $\mathrm{PtFe}$ at $T=0$ is second order. A detailed study of the critical behaviour as a function of concentration will be much more difficult than that as a function of temperature at a normal transition. A number of theoretical models have been developed for ferromagnetic to spin glass transitions [8-11]. Second order transitions seem to be generally favoured. Ordering temperature data on a ferromagnetic to spin glass transition in Fe-Mn amorphous alloys have been analysed using the theory developed for a spin-flip phase diagram [12]. It is not clear that this type of analysis is appropriate if the transition between the two states at $T=0$ is second order and not first order.

A second experimental point appears clearly in the data. For each of the two systems, the field necessary to saturate the $T=0$ magnetoresistance (i.e. to fully align the spins) is practically concentration independent over the range of concentration we have covered, figure 5. These fields are of the order of $10 \mathrm{kG}$ for $\underline{\mathrm{PdCo}}$ and $30 \mathrm{kG}$ for $\underline{\mathrm{PtFe}}$. We can give a

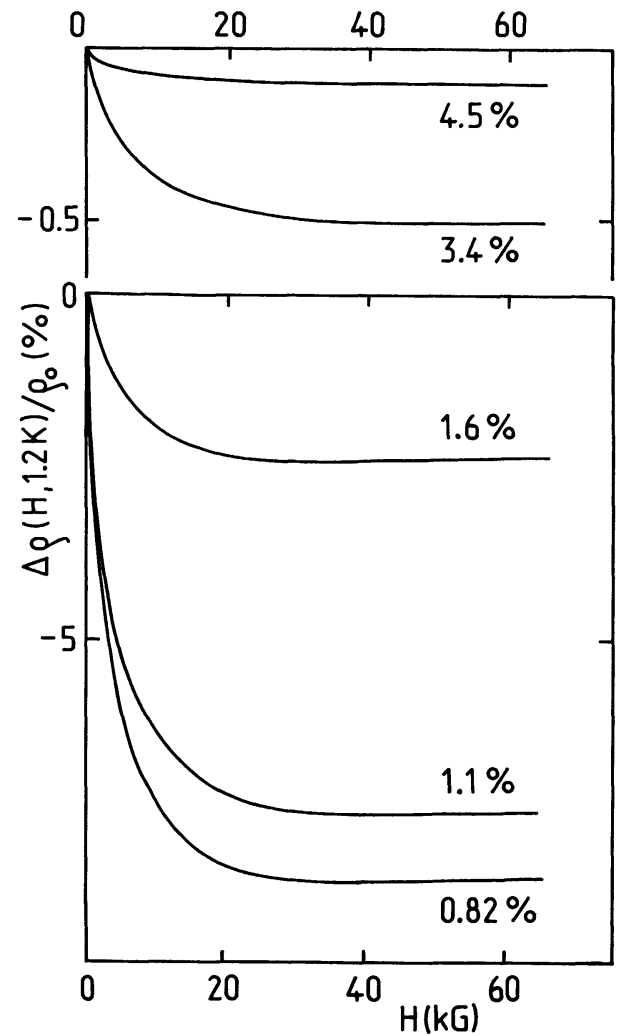

Fig. 5. - The magnetoresistance $\Delta \rho / \rho_{0}$ at $1.2 \mathrm{~K}$ in $\mathrm{PtFe}$ as a function of concentration. Small ordinary magnetoresistance corrections have been made. schematic discussion of the energy terms in play. If we consider each spin submitted to a ferromagnetic ordering interaction $E_{\mathrm{F}}$, and a fraction $\eta$ of the spins submitted to a random disaligning interaction $E_{\mathrm{dis}}$, then $E_{\mathrm{F}} \simeq k T_{\mathrm{c}}$ and $E_{\mathrm{dis}} \simeq \mu H_{\mathrm{s}}$ where $H_{\mathrm{s}}$ is the field required to saturate the magnetization at $T=0$. At a given concentration the average magnetization per atom for $H=0, T=0$ will be

$$
\langle M\rangle^{0} / S \simeq 1-\frac{\eta E_{\mathrm{dis}}}{k T_{\mathrm{c}}} .
$$

For the two cases we consider it appears that $T_{\mathrm{c}}$ is roughly proportional to the concentration while $E_{\mathrm{dis}}$ is practically concentration independent over the concentration range we have studied. As the concentration is reduced we will pass from a ferromagnetic regime to a spin glass regime when $T_{\mathrm{c}}$ drops to a value such that $k T_{\mathrm{c}} \simeq \eta E_{\mathrm{dis}}$.

This fits the observed behaviour if we choose $\eta \simeq 1$, i.e. each spin is subject to a weak random disaligning effect.

There appears to be a clear analogy between the observed breakdown of ferromagnetic order as the ratio $E_{\mathrm{dis}} / E_{\mathrm{F}}$ increases and the breakdown of ferromagnetic order in a standard ferromagnet with temperature as $T / T_{\mathrm{c}}$ increases. At low values of the ratio, we have suggested [13] that the slightly disaligned state can be understood in terms of frozen-in magnons; as the critical point is reached we have a second order transition for the parameter $\langle M\rangle$. There is however an obvious distinction in that for a standard ferromagnet \langle\rangle is a time average while here we have a space average.

We can note in passing that for a number of systems of this type a phase diagram has been suggested in which at fixed concentration the ferromagnetic state transforms to a spin glass state at low temperatures $[14,1]$. Resistance measurements in zero field rule this out : if a spin glass state set in at a low temperature, the resistivity would increase as $T$ dropped, but no such effect is observed experimentally [15]. However the quasiferromagnetic character of these alloys near the critical concentration (which we can expect to be general in all these systems) may explain the behaviour. For instance, when spins are not fully aligned, rotation of the overall magnetization can be hindered by the Dzyaloshinsky-Moriya mechanism [16] which is proportional to $\mathbf{S}_{\mathbf{i}} \wedge \mathbf{S}_{\mathrm{j}}$; this effect is obviously not operative for a true ferromagnet but can restrict the initial susceptibility of a quasiferromagnet. The drop in initial susceptibility at low temperature, which was taken as signaling a spin glass, does not indicate any change in the local spin disorder but just a limited freedom of rotation in applied fields.

We have not so far mentioned the mechanism for the disalignment. For some systems the disalignment is clearly due to antiferromagnetic interactions between certain pairs of spins. Thus in PdMn for 
$c<4 \%$ Mn the system is a quasiferromagnet with $T_{\mathrm{c}} \sim 5 \mathrm{~K}$, but the magnetization can only be fully saturated at fields greater than $200 \mathrm{kG}[17,13]$. This indicates the presence of a relatively low fraction of strong antiferromagnetic couplings, presumably between those Mn spins which are near neighbour to each other [17]. For the PtFe and PdCo on the other hand, the disalignment energies are very weak (of the order of 50 times weaker than in PdMn) but appear to apply to the majority of spins. Two mechanisms suggest themselves : first, the disalignment could represent the effect of magnetic interactions with random sign between spins at rather large distances (while the interactions between nearer spins are all ferromagnetic). Alternatively orbital moments on the impurities could couple into weak random local crystal fields. We have argued [13] that the absence of evidence for disalignment effects down to concentrations of $0.15 \% \mathrm{Fe}$ in $\mathrm{PdFe}$ (where there is no local orbital moment) indicates that the PdCo disalignment has orbital origin. Either mechanism could be invoked for the PtFe case.

To summarize, we have shown that in $\mathrm{PtFe}$ at $T=0$ there is a second order transition from quasiferromagnetic to spin glass as a function of concentration at a critical concentration of about $0.9 \% \mathrm{Fe}$. In PdCo similar behaviour appears to occur with a critical concentration of less than $0.1 \%$ Co. In both cases the ferromagnetic state is gradually broken down by disalignment effects over a wide concentration range above the critical concentration. We expect to find similar behaviour in other systems.

\section{References}

[1] Coles, B. R., Sarkissian, B. V. B. and TAYlor, R. M., Philos. Mag. B 37 (1978) 489.

[2] Star, W. M., Foner, S. and McNiff, E. J., Phys. Rev. B 12 (1975) 2690.

[3] Yoshida, K., Phys. Rev. 107 (1957) 396.

[4] Crangle, J. and Scott, W. R., J. Appl. Phys. 36 (1965) 921.

[5] Loram, J. W., White, R. J. and Grassie, A. D. C., Phys. Rev. B 5 (1972) 3659.

[6] Ododo, J. C., J. Phys. F : Metal Phys. 9 (1979) 1441.

[7] Williams, G. and Loram, J. W., J. Phys. Chem. Solids 30 (1969) 1827 ;

Grassie, A. D. C., Swallow, G. A., Williams, G. and Loram, J. W., Phys. Rev. B 3 (1971) 4154.

[8] Matsubara, F. and Sakata, M., Prog. Theor. Phys. 55 (1976) 672.

[9] Binder, K., Kinzel, W. and Stauffer, D., Z. Phys. B 36 (1979) 161.
[10] Shuka, P. and Wortis, M., Phys. Rev. B 21 (1980) 159.

[11] Fishman, S. and Aharony, A., Phys. Rev. B 21 (1980)280.

[12] Salomon, M. B., Rao, K. V. and Chen, H. S., Phys. Rev. Lett. 44 (1980) 596.

[13] Hamzić, A., Senoussi, S. and Campbell, I. A., J. Phys. F : Metal Phys. 10 (1980) L 165.

[14] Veerbeck, B. H., Nieuwenhuys, G. J., Stocker, H. and Mydosh, J. A., Phys. Rev. Lett. 40 (1978) 586 ;

Carnegie, D. W. and Claus, H., Phys. Rev. B 20 (1979) 1280 ;

Mydosh, J. A., Nieuwenhuys, G. J. and VeerbeCK, B. H., Phys. Rev. B 20 (1979) 1282.

[15] Senoussi, S., Hamzić, A. and Campbell, I. A., J. Phys. F : Metal Phys. 10 (1980) 1223.

[16] Fert, A. and Levy, P. M., Phys. Rev. Lett. 44 (1980) 1538.

[17] Smit, J. J., Nieuwenhuys, G. J. and De Jongh, L. J., Solid State Commun. 30 (1979) 243.

[18] Nieuwenhuys, G. J., Phys. Lett. 67A (1978) 237. 